\title{
Democracia y participación comunitaria en los servicios de salud
}

\begin{abstract}
RESUMEN
El artículo da cuenta de la participación comunitaria en la cogestión de los servicios de salud en la Comunidad Local de Administración de Salud (CLAS) San Martín de Porres. El objetivo de la investigación ha sido identificar los logros de la participación ciudadana en relación a la representatividad, elemento principal en la democratización y descentralización de los servicios de salud en el Perú. La metodología comprende: observación participante, encuesta a 107 pobladores del asentamiento humano mediante cuestionario, entrevista estructurada a 3 presidentes de la CLAS San Martín de Porres y análisis de experiencias a nivel nacional. Los resultados muestran que la política focalizada, implementada por el Estado desde 1994, no promociona la participación democrática de la población en los servicios de salud. La participación es controlada y dirigida a buscar eficiencia y equidad en la gestión de los servicios de salud, mas no el ejercicio democrático como finalidad. En la práctica se constata que la forma como ha sido diseñada la política de salud no garantiza dicha participación.
\end{abstract}

Palabras clave: participación, democracia, salud, focalización, ClAS.

\section{Democracy and community participation in health services}

\begin{abstract}
The article gives an account of community participation in the co-management of health services in the Local Community of Health Administration (CLAS) San Martin de Porres. The objective of the research has been to identify the achievements of citizen participation in relation to representativeness, a major element in the democratization and decentralization of health services in Peru. The methodology includes: participating observation, survey of 107 people of the human settlement by questionnaire, structured interview with 3 presidents of the CLAS San Martin de Porres and analysis of experiences at the national level. The results show that the targeted policy, implemented by the State since 1994, does not promote the democratic participation of the population in health services. Participation is controlled and aimed at seeking efficiency and equity in the management of health services, rather than democratic exercise as a purpose. In practice, it is found that the way in which health policy has been designed does not guarantee such participation.
\end{abstract}

KeYwords: participation, democracy, health, focus, CLAS. 


\section{Introducción}

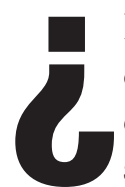
Por qué es importante la participación ciudadana en los servicios de salud? Investigar esta forma de participación en los servicios de salud, particularmente la representatividad como uno de los elementos esenciales de la democracia, encuentra su relevancia en un contexto donde estos servicios no se brindan con la calidad que ameritan. Por tanto, la participación de la población ya no puede ser un medio utilizado por el Estado como instrumento para alcanzar objetivos institucionales. Hace más de cuarenta años la Declaración de Alma Ata reconoció la participación de la comunidad como estrategia principal para mejorar la salud primaria en los siguientes términos:

La atención primaria sanitaria requiere y promociona un autodesarrollo comunitario e individual al máximo posible, con participación en la planificación, organización, desarrollo y control de la atención primaria sanitaria, haciendo un uso más completo de los recursos locales y nacionales y de otros recursos disponibles; y para finalizar, desarrolla, a través de una formación apropiada, la habilidad de las comunidades para participar. (Salud Pública de México, 1978)

En el Perú, la política focalizada de salud se da en un contexto de descentralización y democratización del Estado; a partir de entonces (1994) el Ministerio de Salud (MINSA) incorpora la participación ciudadana en el diseño e implementación de políticas públicas de salud, involucrando a la comunidad a través de sus representantes elegidos democráticamente ante la CLAS, quienes tienen la obligación de mantener informada a la población sobre la gestión así como llevar los acuerdos y objetivos de la comunidad para ser defendidos en la Directiva, donde participan, a través de sus legítimos derechos respaldados por una frondosa normatividad nacional e internacional.

Aquí pretendemos describir cómo se viene cumpliendo la participación ciudadana respecto a la representatividad de la comunidad, dado que miembros de la misma vienen participando más de 18 ańos aproximadamente. Sin embargo, hay estudios referidos a otras CLAS donde se evidencia una participación controlada y dependiente, factor que obstaculiza su desarrollo. Elegimos el problema de la representatividad de la comunidad porque observamos que la política focalizada en salud, diseñada e implementada desde 1994, no cumplía con promover y ejercitar la participación democrática de la comunidad tal como declaraba en los documentos oficiales. Es decir, no se aplican mecanismos democráticos pese a que el modelo de cogestión señala que esta propuesta tiene como objetivo democratizar los servicios de salud. Sobre el tema se han venido realizando algunos estudios de evaluación de esta política, priorizando la eficiencia, equidad y cobertura de los servicios de salud en las zonas rurales; sobre la representatividad, como elemento básico de la participación, solo se ha tocado dentro de otras variables y muy pocas veces.

En un artículo que escribe Portocarrero (2006) sobre la comunidad Laura Caller Iberico, al referirse a la participación democrática de esta, señala que, de los siete miembros de la asamblea de la CLAS, tres son elegidos por la comunidad, luego el médico jefe, que representa al Estado, elige a su criterio a tres pobladores de la comunidad. Además, se le impone a la comunidad a elegir como representantes solamente a aquellos que han tenido cargos como dirigentes (p.177). Este modelo de cogestión todavía existe, sobre todo en las zonas rurales donde los problemas de representación serían mayores; estas formas de implementar políticas de salud no garantizan los derechos ciudadanos que asisten a la comunidad.

\section{Participación comunitaria}

La hoy llamada participación comunitaria, cuando nos referimos a grupos de personas en actitud de autoayuda, es una constante en la historia que se da en momentos donde el ser humano afronta problemas difíciles de resolver individualmente. Sobre todo en los grupos más vulnerables socialmente, las necesidades básicas y su solución son factores que explican la participación; inicialmente frente a situaciones rudimentarias donde la misma naturaleza hacía difícil obtener los satisfactores de sobrevivencia. Desde el inicio de la cultura occidental, la participación se mantiene como forma de organización para la ayuda mutua entre los más necesitados, en sociedades de profunda desigualdad social y ulteriormente en países dependientes y, por tanto, no desarrollados (Zakus, 1988, p. 156). 
Otra es la participación social como factor importante en la implementación de los servicios públicos que presta el Estado, por ejemplo: salud, educación, vivienda, saneamiento físico, entre otros. Esta forma de participar ha venido evolucionando producto de los cambios económicos, políticos y sociales, así como de las perspectivas y grados de desarrollo; particularmente del tipo de políticas públicas, sociales y objetivos que estas tengan respecto al bienestar de la población. Así, por ejemplo, la participación en el modelo desarrollista no será igual a la que se da en el modelo neoliberal, porque aun cuando tengan algunos elementos comunes, las necesidades y formas de atenderlos corresponden a contextos distintos.

En las décadas 60 y 70 del siglo pasado, toda América Latina utilizó la estrategia del desarrollo comunitario con el objetivo de incluir a grupos considerados al margen del desarrollo social y económico (Gómez, 2008, p.2); para ello, las instituciones públicas y privadas se volcaron hacia las poblaciones vulnerables socialmente, que ocupaban las zonas rurales y urbanas, buscando educar, capacitar e incluso concientizar sobre la realidad en pro de impulsar su desarrollo. Entre las perspectivas de desarrollo destacó una que tenía como propósito contribuir a un cambio social que se inicie desde las poblaciones más necesitadas. Para alcanzar dicho propósito, había que educar a las personas para que conozcan sus derechos y el lugar que ocupan en la sociedad; esperando que ellos mismos cambien su situación construyendo una sociedad más justa e igualitaria, con libertad para los oprimidos. En esta perspectiva destacó el filósofo y pedagogo brasileño Paulo Freire, asesor de la UNESCO, institución que reconoció este método y recomendó su aplicación en la alfabetización de las masas populares; otorgándole el premio «Educación para la Paz» en 1992. En ese mismo año Freire recibió el premio «Andrés Bello» otorgado por la Organización de los Estados Americanos en reconocimiento a su labor como educador (Ocampo, 2008, p. 62).

En su obra "Pedagogía del oprimido», el educador Freire dice que las masas oprimidas deben tener conciencia de su realidad y deben comprometerse, en la praxis, para su transformación. En ello tiene gran relevancia la educación, pues la pedagogía del oprimido busca crear conciencia en las masas oprimidas para su liberación. La alfabetización del oprimido debe servir para enseñarle no solamente las letras, las palabras y las frases, sino lo más importante, «la transmisión de su realidad y la creación de una conciencia de liberación para su transformación en un hombre nuevo" (Ocampo, 2008, p. 63).

\section{Participación comunitaria y democracia en las políticas de salud}

A partir de los años 80 del siglo pasado en América Latina, y los 90 en Perú, se diseñan e implementan las políticas sociales focalizadas en salud, acordes con el reordenamiento económico mundial. Aquí se considera la participación ciudadana como estrategia para la mejora de los servicios de salud; especialmente en nuestra región. Desde entonces, los científicos sociales han asumido miradas críticas sobre el sistema y las formas de desarrollo que propugna; se propuso un cambio social a partir de acciones que traspasaron las fronteras profesionales. En este nuevo contexto, la participación comunitaria incluye conceptos como los de ciudadanía, igualdad y libertad.

Como se expuso con anterioridad, el tema de la búsqueda del desarrollo constante, incluir parámetros de modernización y la necesidad de una sociedad movilizada pero despolitizada requería, a la vez, de un profesional capaz de trabajar con las personas para «enseñarles» las bondades de ser moderno y «demostrarles en que se equivocaban». (Bettina, 2005, p.195)

Este tipo de participación es muy distinto a la participación donde media la implementación de un programa de educación, salud u otros servicios vinculados directamente con el Estado y el desarrollo de cada país.

Como se constata, la participación comunitaria, en esta etapa, tiene un objetivo concreto: atender las necesidades básicas de todo individuo y familia. Es una participación que busca agenciarse de recursos y mejores formas de mantener o recuperar su salud mediante su propio involucramiento; esto frente a un Estado carente de medios económicos para una atención eficiente y eficaz, reclamada por la población. La política focalizada tenía como meta, a corto plazo, mejorar los servicios de salud; para ello una de sus estrategias fue agenciarse de recursos financieros 
y humanos mediante la participación comunitaria; que, según el discurso del Estado, en adelante sería una participación democrática. La realidad ha sido distinta, solo en contados países se ha creado mecanismos para empoderar a la población, respetando su autonomía y libertad como por ejemplo en Cuba, Brasil, Colombia y Bolivia; entre los pocos de América Latina.

Tenemos entonces que, de acuerdo a las formas de ejercicio de la democracia y a los paradigmas sociales como concepto y como práctica, existen variados puntos de vista sobre la participación social. Un concepto de participación basado en derechos dice, por ejemplo, lo siguiente:

La participación es un componente esencial de la democracia, como forma de organización social y de gobierno. En las ciudades existen diferentes formas de participación: social, comunitaria, ciudadana, política. Todas son necesarias para hacer de los individuos que la habitan ciudadanos con derechos y obligaciones sociales, políticos, urbanos. (Ziccardi, 1999, pp. 2-3)

Por su parte, Briceño (1998) hace una crítica a la participación vinculada al Estado, como complemento de las acciones sociales, donde este no está en la capacidad de dar respuestas eficientes a las necesidades de la población. Así, bajo el concepto de participación democrática lo que hace es disminuir los costos del servicio o simplemente hacer que los individuos paguen directamente de esa forma los servicios y que el Estado se sustraiga de su responsabilidad. Este mismo autor también nos muestra otro tipo de participación; cuando la comunidad se organiza contra el poder para criticar la calidad de los servicios, las políticas sociales, las estrategias, o cuando los funcionarios los tratan como ignorantes (Briceño, 1998, pp. 4-5).

Otra forma de participación como fin, contraria a la anterior, se fundamenta en las necesidades de cada realidad, tiene objetivos propios, une más a la comunidad y va más allá de cualquier proyecto o política para contribuir al desarrollo sostenible. Esta es una participación directa que influye desde abajo para determinar las prioridades e introducir cambios en las instituciones y en las políticas sociales, las que deben nacer de la propia comunidad y no ser impuestas desde afuera. Este autor dice:
Es una modalidad intrínsecamente superior de participación, los participantes cuentan con algunas atribuciones, que van desde el derecho a imponer su veto temporal o permanente al de participar de modo directo en el proceso decisorio... las relaciones entre quienes deciden y los afectados por sus decisiones pueden dar lugar a muy diversas formas de participación. (Oakley, 1990, pp. 10-11)

Si debemos resumir de algún modo la participación comunitaria, concluiríamos señalando que hay dos formas básicas con características fundamentales: a) Se trata de un conjunto de actividades que empoderan a la comunidad identificando sus necesidades, proponiendo alternativas de solución y luchando por alcanzar sus objetivos. Es una forma independiente que ejerce su derecho a la salud, por tanto, democrática pero no contradictoria al Estado. b) Cumple actividades con la dirección vertical de los gobernantes para abaratar los costos de los servicios y contribuir con su trabajo en la ejecución de políticas de salud; diseñadas incluso fuera del propio país. Esta es una participación controlada por el Estado, sin visos de democracia.

\section{Participación democrática pobreza y condicionamientos externos}

En la Declaración de Alma Ata (1978) se proyectó la meta «Salud para todos en el año 2000», donde se asumió que los problemas de salud en América Latina y otros países atrasados eran producto de su limitado desarrollo económico. Para mejorar dichos servicios se priorizó la promoción y prevención en el nivel de atención primaria y la participación comunitaria, como elemento básico para alcanzar los objetivos en los programas de salud. En varios Estados de América Latina se venían implementando algunas estrategias recomendadas, sobre todo la atención a los grupos olvidados de las zonas rurales y urbanas donde habitan poblaciones pobres y pobres extremos. Lo que se acordó en dicho evento fue un preludio de los acontecimientos vividos en la década de los 80, con los lineamientos del neoliberalismo impuestos en nuestros países, en crisis y con ingentes deudas. El discurso neoliberal en lo social fue claro respecto al papel del Estado; no más sería protector social y distribuidor de la riqueza mediante las 
políticas sociales. En adelante, las funciones de este serían mínimas: en lo social, solo atendiendo las necesidades de las poblaciones llamadas desde entonces «pobres extremos»; en lo económico, ajustado a las imposiciones del Fondo Monetario Internacional (FMI) y al servicio del mercado.

Las políticas focalizadas en salud en general para América Latina responden a uno de los requerimientos del modelo económico neoliberal, el cual consiste en reducir los gastos sociales para este sector y dirigir la atención solo a los pobres extremos. Las institucio- nes encargadas de implementar estos cambios, que favorecen a los grandes capitalistas de los países desarrollados, fueron el Banco Mundial (BM) y el FMI. En adelante el Estado solo debía encargarse de regular el movimiento económico, es decir promover la libre competencia, abriendo las puertas a los grandes capitales foráneos; aun cuando esta se dé en condiciones desiguales entre grandes empresas trasnacionales y pequeñas empresas de los países atrasados, dejando así que el libre mercado sea el regulador de toda la actividad económica.

TABLA 1

Cuadro comparativo sobre el gasto social de diferentes países

\begin{tabular}{|c|c|c|c|c|c|c|c|c|c|}
\hline OCDE & 1980 & 1990 & 2000 & 2010 & CEPAL & 1980 & 1990 & 2000 & 2010 \\
\hline Australia & 10.3 & 13.1 & 17.3 & 16.6 & Argentina & 16.1 & 18.57 & 21.43 & 27.78 \\
\hline Austria & 22.4 & 23.8 & 26.7 & 28.9 & Bolivia & 6.7 & 4.2 & 16.36 & 18.42 \\
\hline Bélgica & 23.5 & 24.9 & 25.4 & 29.4 & Brasil & 14 & 17.63 & 21.15 & 27.06 \\
\hline Canadá & 13.7 & 18.1 & 16.5 & 19.3 & Chile & 17.6 & 11.88 & 14.99 & 15.64 \\
\hline Dinamarca & 24.8 & 25.1 & 25.7 & 30.1 & Colombia & 7.6 & 6.04 & 10.78 & 13.62 \\
\hline Finlandia & 18.1 & 24.3 & 24.3 & 29.1 & Costa Rica & 16 & 16.13 & 17.32 & 22.93 \\
\hline Francia & 20.8 & 24.9 & 27.7 & 31 & Ecuador & 6.12 & 3.95 & 2.86 & 9.84 \\
\hline Alemania & 22.1 & 21.7 & 26.6 & 27.3 & El Salvador & 9.1 & 5.6 & 9.24 & 13.01 \\
\hline Grecia & 10.2 & 16.5 & 19.2 & 23.2 & Guatemala & 6 & 3.87 & 6.54 & 8.07 \\
\hline Irlanda & 16.7 & 14.9 & 13.3 & 22.8 & Honduras & - & 6.44 & 7.82 & 12.04 \\
\hline Italia & 18 & 19.9 & 23.3 & 27.5 & México & 8.6 & 5.53 & 8.62 & 11.29 \\
\hline Japón & 10.4 & 11.3 & 16.5 & 19 & Nicaragua & 10 & 6.98 & 8.6 & 13.00 \\
\hline Luxemburgo & 20.6 & 19.1 & 19.8 & 23.5 & Panamá & 15 & 6.93 & 9.06 & 10.53 \\
\hline Holanda & 24.8 & 25.6 & 19.8 & 22.6 & Paraguay & 2.9 & 2.86 & 9.81 & 11.01 \\
\hline Nueva Zelanda & 17 & 21.5 & 19.1 & 21.8 & Perú & 4.56 & 3.97 & 8.62 & 9.95 \\
\hline Noruega & 16.9 & 22.3 & 21.3 & 24 & Uruguay & 13.7 & 16.35 & 20.69 & 23.32 \\
\hline Portugal & 9.9 & 12.5 & 18.9 & 26.1 & Venezuela & 11.5 & 7.81 & 11.03 & 13.51 \\
\hline España & 15.5 & 19.9 & 20.4 & 26.7 & & & & & \\
\hline Suecia & 27.2 & 30.2 & 28.4 & 28.2 & & & & & \\
\hline Suiza & 13.8 & 13.5 & 17.8 & 19.6 & & & & & \\
\hline Reino Unido & 16.5 & 16.7 & 18.6 & 24.4 & & & & & \\
\hline $\begin{array}{c}\text { Estados Uni- } \\
\text { dos }\end{array}$ & 13.2 & 13.5 & 14.5 & 20.4 & & & & & \\
\hline Promedio & 16.94 & 17.49 & 18.93 & 22.40 & & 10.35 & 8.51 & 12.05 & 15.35 \\
\hline $\begin{array}{c}\text { Coeficiente de } \\
\text { Variación }\end{array}$ & 0.3484 & 0.3898 & 0.3020 & 0.2517 & & 0.4437 & 0.6310 & 0.4605 & 0.4059 \\
\hline
\end{tabular}

Gasto social como porcentaje del PIB entre 1980 y 2010 - Países de la OCDE y América Latina.

Fuente: «Determinantes del gasto social en América Latina». Martín-Mayoral, Espinosa y Nabernegg (2014) ${ }^{1}$

1 En el ańo 2017, el presupuesto ejecutado en el Perú por el Gobierno Central en reducción de pobreza, extrema pobreza y programas de inclusión social representó el 9.5\% respecto al PBI (Cuenta General de la República, 2017, p.410). Para el sector salud el presupuesto en este mismo ańo asciende a 13.776 millones de soles (US\$4.037 millones), lo que representa el 2\% del PBI. 
En lo social, el neoliberalismo significa la sustitución del Estado de bienestar para los países desarrollados y del Estado interventor para los atrasados (Huerta, 2005, p. 15). Cabe resaltar que en los primeros la redistribución económica permite resolver las necesidades materiales de la población, la pobreza en estos países nunca ha tenido ni tiene la magnitud como se muestra en nuestra región. La explicación es simple: el nivel de desarrollo es distinto y los problemas sociales también; para el Perú el bienestar es un tema que no parece preocupar a los gobernantes, somos uno de los tres últimos países que invierten menos en salud y educación.

En nuestro caso, sumado al atraso está la corrupción, el clientelismo, el racismo, la exclusión, entre otros factores que agravan la situación. Cuando nos referimos, entonces, al estado de bienestar, y en primer lugar a la salud, no podemos dejar de considerar las diferencias antes mencionadas. Respecto al estado mínimo, consecuentemente siguen existiendo esas diferencias entre países atrasados y desarrollados; pero también es cierto que entre los primeros también existen diferencias tanto en el estado de bienestar como en el estado mínimo. Lo podemos constatar precisamente en el nivel de gasto social que presentan los diversos países. Lamentablemente el Perú es uno de los tres últimos con menor gasto social, en salud no llega al 3\% de PBI.

Las políticas focalizadas en el Perú son aquellas diseñadas e implementadas desde la década de los 90 por el estado mínimo. Estas han evolucionado en algunos aspectos, pero conservando sus características iniciales tales como: materializar los objetivos del sistema poniendo en práctica los mandatos del sistema capitalista, donde la salud y la educación pasan de ser bienes sociales a mercancías sujetas al libre mercado, y el gasto social debe reducirse en aras del cumplimiento del ajuste fiscal que prioriza el pago de la deuda externa. Los pocos recursos deben ser empleados para la atención de los estratos pobres y pobres extremos. Laurell (1994) describe así la propuesta del BM, en el contexto de su comentario al «Informe sobre el Desarrollo Mundial 1993: Invertir en salud»:

La primera premisa subyacente al documento es que la salud pertenece al ámbito privado. Por tanto, el Estado-Gobierno solo debe ocuparse de los problemas de salud cuando los privados no quieren o pueden resolverlos. A esta premisa se añade otra que se resume en que el sector público es ineficiente y frecuentemente inequitativo - debido a las diferencias de poder de presión sobre el gobierno de los distintos grupos - mientras que el sector privado es eficiente y más equitativo ya que obedece a la competencia y elección libres en el mercado.

Asimismo, el neoliberalismo, a través del BM, sostiene y expresa la concepción individualista respecto a los servicios de salud cuando asevera que lo más importante es lo que el individuo y su familia hagan por su salud y no lo que los gobiernos hagan por él.

Es decir, la salud-enfermedad del individuo no es producto de su condición económica y social alguna y este no pertenece a una sociedad, por tanto, solo él es responsable de su salud. De esta forma se descarga la responsabilidad de la salud en la familia, pero principalmente en la mujer de los sectores empobrecidos, que es la llamada a participar en los servicios de salud sin ninguna remuneración. Así, el Estado reduce el gasto social en ese sector. (Laurell, 1994, p. 3)

La focalización para los servicios de salud en el Perú ha considerado la participación comunitaria como un elemento fundamental para lograr la equidad, eficiencia y eficacia de los mismos. Sin embargo, su incorporación a estos ha demostrado que los verdaderos objetivos se sustentan en el cumplimiento de las exigencias del BM y el FMI, las cuales se traducen en la reducción de los gastos en salud para la población en mayor riesgo social y responder de alguna forma al clamor popular, debido a los altos índices de pobreza después del Fujishock y la pésima situación de los centros y puestos de salud por la crisis económica.

\section{Democracia y participación comunitaria en la CLAS San Martín de Porres}

El caso estudiado tiene pocos antecedentes, a pesar de que hasta la actualidad es vigente en la atención primaria de salud. Los Comités Locales de Administración Compartida en Salud (CLAS), hoy denominados Comunidad Local de Administración de Salud (CLAS), fueron creados por el Decreto 
Supremo 01-94-SA promulgado el 5 de mayo de 1994 durante el gobierno de Alberto Fujimori. El Programa de Administración Compartida de los Servicios de Salud (PAC) propuso esta forma de organización social, que luego se oficializó, donde la comunidad participaría por primera vez como cogestora de dichos servicios. Estas organizaciones siguen funcionando, aunque con modificaciones que se han venido dando en sus más de 20 años de creación, pero manteniendo muchas de sus características hasta la actualidad.

Nuestro interés es develar en qué sentido esta forma de participar es democrática, toda vez que ha sido creada en un contexto de descentralización y Estado de Derecho. De acuerdo con los documentos del Ministerio de Salud (MINSA), un CLAS es:

[...] una asociación civil, con personería jurídica, integrada por representantes de la comunidad organizada, para administrar un puesto o centro de salud, con el fin de mejorar la salud de la comunidad. Es una modalidad descentralizada de administración, donde el Estado comparte esfuerzos y recursos con la comunidad. (MINSA, 1996, p.15)

De este modo, la política focalizada crea una nueva relación Estado-comunidad mediante un contrato de carácter privado de acuerdo al Código Civil, donde el objeto de contratación es un programa o plan local de salud que la CLAS se compromete en su elaboración y ejecución.

La nueva política en salud involucra la participación de la comunidad con los objetivos de ampliar la cobertura de atención con eficiencia y equidad. Además, establecer un control más cercano de los servicios de salud a través los mismos usuarios. El modelo de cogestión CLAS es concebido, diseñado y puesto en marcha solo por el Estado, y la forma de participación, en este caso, es concretamente para la elaboración y ejecución del plan de salud con las características definidas desde el MINSA. La comunidad no es convocada en ningún momento para participar en la creación del modelo de gestión, donde ya está definida la forma de su participación que además solo está considerada para la gestión de los servicios de salud. Por ejemplo, la elección de los representantes de la comunidad venía indicada para realizarse del siguiente modo:
TABLA 2

Elección de los representantes de la Junta Directiva de la CLAS

\begin{tabular}{|c|c|}
\hline ELECTORES & ELEGIDOS \\
\hline $\begin{array}{c}\text { Jefe del establecimiento ante la } \\
\text { Dirección Subregional de salud }\end{array}$ & $\begin{array}{c}3 \text { miembros de la } \\
\text { comunidad }\end{array}$ \\
\hline $\begin{array}{c}\text { Las organizaciones de base (comedores, } \\
\text { comités de salud, vaso de leche) }\end{array}$ & $\begin{array}{c}3 \text { miembros de la } \\
\text { comunidad }\end{array}$ \\
\hline Ministerio de Salud & $\begin{array}{c}1 \text { médico jefe como } \\
\text { miembro nato }\end{array}$ \\
\hline
\end{tabular}

Elaboración propia

Veamos cómo se eligen a los representantes de la comunidad. Tres representantes son elegidos entre los comités de salud, comedores populares y otras organizaciones; no por toda la comunidad. Otros tres, que también son miembros de la comunidad, pero son elegidos, para representar al Estado, por el médico jefe del establecimiento; en coordinación con la Subregión de Salud; al final, el médico jefe se incorpora como miembro nato.

De acuerdo a este método de elección, habría cuatro miembros que representan al Estado, siendo tres de la comunidad y uno del MINSA, y tres que representan a la comunidad. Es decir, la estructura de poder está clara, la mayoría representa al Estado. Lo que llama la atención es que se elijan dentro de la comunidad a personas que van a representar al Estado, los otros tres miembros restantes no son elegidos por asamblea general de la comunidad sino por las organizaciones de base que de ningún modo pueden sustituir a toda la comunidad. Esta forma de elección es totalmente arbitraria y no garantiza una participación democrática de la comunidad.

Respecto a la particularidad de la política en mención, hay ex funcionarios y estudiosos convencidos de que esta forma de gestionar los servicios de salud responde a momentos muy complejos en el Perú. En 1994 el país estaba devastado por acontecimientos como la crisis económica de los 80 , la lucha armada de Sendero Luminoso y el Fujishock que afectaron sobremanera, aumentando la pobreza, el descontento de la población y el casi colapso de los puestos y centros de salud en el país.

Los factores que afectaron al Perú no solo fueron internos sino también las exigencias de aplicar los lineamientos impuestos por los organismos internacionales (BM y FMI) respecto a la reducción del gas- 
to social por parte del Estado. Frente a ello se buscó revertir esta situación, encontrando la forma de mejorar los servicios de salud que involucraban a los más pobres. Frisancho (2005) expone así la experiencia de las CLAS en Perú:

Además, las limitaciones del modelo tradicional de gestión pública de los servicios del primer nivel encontraban dos aportes innovadores: el control social e intervención directa de los ciudadanos y la incorporación de normas del régimen laboral privado para superar los problemas de contratación y desempeño. (p.6)

Otros expertos como Díaz (2001) consideran que la propuesta CLAS se da en un contexto de descentralización, nuevas formas de financiamiento y con participación que significan un avance en relación a las políticas tradicionales que eran verticales. Esta incorporaba a la comunidad por primera vez en la gestión de recursos públicos. Por tanto, todos los nuevos mecanismos están articulados para su funcionamiento adecuado. Finalmente, resalta las bondades del modelo como innovación donde la comunidad sí tiene poder de decisión, como nunca antes lo había tenido.

Arroyo (2001) realizó un estudio sobre la política de salud en la década de los 90, sobre las CLAS en general. Una de sus conclusiones fue que la participación no tuvo mayor alcance, es más:

[...] no encontró diferencias relevantes sobre la información que tenía la ciudadanía respecto a los servicios de salud anotando que solo el 43,9\% de entrevistados conocía los horarios de atención, $38,7 \%$ había participado de alguna reunión informativa, el $40 \%$ de los líderes de la comunidad no sabían sobre la existencia de la CLAS, y solo un $38,8 \%$ de los líderes habían sido consultados sobre asuntos de la CLAS. (p. 46)

Sobre la elección y representatividad, se reitera la forma de elegir a representantes de la comunidad mediante estudios realizados en algunas regiones del país. Se ha verificado que los representantes no son elegidos en asambleas generales de la población, sino son personas voluntarias, representantes de organizaciones. Por lo tanto, estamos en una representatividad informal o no existe tal representatividad. Cortez y Phumpiu (1999) realizaron un estudio sobre la repre- sentatividad en las subregiones de Lima, La Libertad y Junín y concluyen que:

En promedio, el $80.8 \%$ de los entrevistados afirmó que no sabía que el establecimiento de salud era administrado conjuntamente por representantes de su comunidad y del Estado. El 95.8\% no participó en la elección de los representantes de la comunidad y el $91.3 \%$ no conocía los nombres de los representantes. Además, el 84.6\% no sabía que los representantes de la comunidad convocan a reuniones periódicas para informar sobre la labor realizada. (p. 30)

Albújar (2016) ha investigado la CLAS de Miraflores Alto en Chimbote y respecto a la participación de la comunidad en la elaboración del Plan Local de Salud presenta este testimonio de una de las enfermeras de la CLAS:

No, ellos (los pobladores) no participan, ellos ven el PSL elaborado, es un trabajo tedioso, mínimo toma 15 días a 3 semanas en elaborarlo, entonces lo que dicen los gerentes y yo les doy la razón, 'que si involucraban a la comunidad, por el nivel cultural nos iba a tomar 2 a 3 meses y no es por desmerecerlos' y con la presión del nivel superior, que amenazan con los sueldos, si no les enviamos la información, peligra los sueldos. Muchas veces lo hemos elaborado (nosotros) hasta muy de madrugada.

Otra enfermera del mismo CLAS responde a la misma pregunta: "Normalmente lo hace 1 o 2 personas y luego lo socializan. Ahora tienen la idea de que cada profesional realice la parte que le corresponde respecto a su área y después se socialice». Un médico responde a la pregunta así: «Han habido algunas incoherencias respecto a la normativa en cuanto a la elaboración PSL. La normativa dice que debe ser elaborado con la comunidad al menos no hemos participado de ello y la comunidad menos». Finalmente, los miembros de la asamblea dicen: «los trabajadores conocen las necesidades más que nosotros» (pp. 68-69).

Las evidencias muestran la realidad donde participan los miembros de la comunidad; el interior de las CLAS varía de acuerdo a factores como: información, sobre todo en lo concerniente a su participación; grado de instrucción; conocimientos informáticos y de gestión; equipos a su disposición; vivir cerca a los centros de salud, etc. Partiendo de estas consideracio- 
nes se presenta una clara diferencia en la participación de la comunidad en zonas urbanas y rurales, en estas últimas las condiciones son mucho más precarias por las características geográficas, económicas, educativas y nivel de instrucción entre otras limitaciones.

Bowyer (2004) ha realizado una investigación sobre las CLAS en comunidades alejadas de Cajamarca y Ayacucho. De acuerdo a sus hallazgos él considera que el modelo es rígido y vertical para una realidad impredecible que requiere más bien de modelos flexibles, adaptables a las circunstancias.

[...] los CLAS funcionan desde un modelo compuesto por definiciones y de roles fijados claramente, las obligaciones, los procedimientos y los métodos impartidos por la autoridad jerárquica que posee un estilo inflexible similar a la gerencia y las oportunidades son restringidas para las formas horizontales de interacción. (p. 7)

Según Bowyer, este modelo construido no permite que las CLAS puedan identificar los factores que pueden hacer factible la participación de las comunidades rurales; o sea una política que limita la participación de la comunidad

\section{Metodología}

La investigación es aplicada, exploratoria y descriptiva por cuanto hay escasas investigaciones sobre este problema. Enfoque cuantitativo, no experimental y transversal. El universo es de 22832 habitantes del asentamiento humano San Martín de Porres, mayores de 18 años. La muestra es de 107 habitantes que fueron elegidos por muestreo probabilístico aleatorio simple. Las unidades de análisis han sido hombres y mujeres de la comunidad mayores de 18 años que se atienden en el centro de salud. Los datos que fundamentan la investigación proceden de fuentes primarias y secundarias; el tipo de datos es cuantitativo. Como técnicas se ha usado la entrevista y la observación participante, y como instrumento, el cuestionario.

\section{Resultados}

Los resultados de la encuesta realizada a la comunidad, diálogos y entrevistas estructuradas a sus dirigentes dan como resultado que no se ejerce participa- ción ciudadana; uno de los factores es que la llamada cogestión es una forma de participación controlada por el Estado toda vez que la forma de elección ya viene diseñada como parte de la política de salud. Es así que a la pregunta "¿conoce usted cómo participa la comunidad en la CLAS?», el $55 \%$ no sabe o no responde, esa es la mejor prueba que la comunidad no elige a sus miembros que participan en la CLAS. La elección que se realiza no se fundamenta en los principios democráticos, por tanto, no hay representatividad. De esta forma se da respuesta al objetivo de identificar el grado de representatividad de la comunidad en la cogestión de los servicios de salud en su jurisdicción.

\section{Discusión de resultados}

La salud es un derecho humano fundamental reconocido en la constitución política del Perú y en múltiples tratados internacionales, por tanto, las políticas sociales no pueden atentar contra la salud de los más necesitados desconociendo sus derechos a la participación en el diseño e implementación de dichas políticas. Como hemos venido argumentando la participación comunitaria en los servicios de salud es considerada en todo el mundo como un factor esencial para atender las necesidades reales que la población demanda. Sin embargo, en nuestro país no se fomenta la participación democrática respetando el espacio de la comunidad, a pesar de que en el discurso político, en las políticas públicas y en una serie de documentos oficiales se ha legalizado este derecho. La Constitución Política del Perú (1993), documento legal que ordena los derechos que tenemos los peruanos, señala en el Capítulo III, Artículo 31:

Los ciudadanos tienen derecho a participar en los asuntos públicos mediante referéndum; iniciativa legislativa; remoción o revocación de autoridades y demanda de rendición de cuentas. Tienen también el derecho de ser elegidos y de elegir libremente a sus representantes, de acuerdo con las condiciones y procedimientos determinados por ley orgánica. Es derecho y deber de los vecinos participar en el gobierno municipal de su jurisdicción. La ley norma y promueve los mecanismos directos e indirectos de su participación... La ley establece los mecanismos para garantizar la neutralidad estatal 
durante los procesos electorales y de participación ciudadana. (1993, p. 12)

Parece que en el diseño de las políticas de salud no se toma en cuenta el sustento legal más importante como es nuestra carta magna. La participación ciudadana en este sector continúa siendo vulnerada al no permitir la independencia y libertad de las poblaciones para proponer soluciones a sus problemas, incidir en las políticas de salud y ejercer un control sobre la implementación de este servicio. Sin bien esta forma auténtica de participación está reconocida oficialmente, nos preguntamos entonces: ¿cuáles son las razones por las que, hasta ahora, en la práctica, sobre todo en los países de nuestra región, la participación sigue siendo un instrumento del Estado para ahorrar costos de implementación y alcanzar los logros esperados en programas y proyectos aplicados verticalmente?

Veamos la experiencia de las CLAS en Perú, reconocida por muchos estudiosos como una forma de participación innovadora que se puso en marcha para los grupos de pobladores pobres y pobres extremos. Se ha dicho también que esta política focalizada se ha dado en el marco no solo de la descentralización, sino también de la democratización en los servicios de salud, donde la participación ciudadana fue considerada como muy importante.

El problema que se planteaba la Autoridad Nacional de Salud era ¿cómo mejorar la gestión de dichos establecimientos de salud, incrementando el acceso y la calidad de los servicios? Esta era una necesidad impostergable, evidenciada de manera dramática por los indicadores de salud, de producción de servicios, de cobertura de atención y de demanda insatisfecha, frente a los que los sistemas tradicionales de organización de los servicios de salud no garantizaban la mejora esperada en su capacidad de respuesta. (Frisancho, 2005, pp. 4-5)

Vemos coherente la respuesta del Estado a la pregunta y al argumento planteado en relación a la situación en la que se encontraban los servicios de salud de los más pobres (producción, cobertura de atención y demanda insatisfecha). La propuesta del MINSA es establecer control social para revertir los indicadores mencionados; pero ¿cómo se garantizaba que se dé efectivamente este control?, ¿qué concepto de control social y forma de ejecutarlo primó en las autoridades del MINSA?

Según Arévalo (2004), en un Estado democrático y por tanto de derecho, el pueblo es soberano y delega el poder a los gobernantes y autoridades; por ello mismo tiene el derecho de controlar, incluso incidir en el diseño de las políticas de salud, más aún en su implementación. La participación como control es necesaria en la rendición de cuentas y visibilidad de la gestión pública; lo es también para el empoderamiento de la comunidad y garantizar el ejercicio democrático. Además, permite el desarrollo de capital social, conocer sus derechos ciudadanos (p.110). El ejercicio de la ciudadanía en su relación con el Estado es un derecho legitimado por los derechos humanos que tienen los individuos y la sociedad en general, considerado como una forma de inmunidad frente al poder del Estado y que este mismo lo garantiza y respalda.

En la experiencia de las CLAS no se especifica cómo, hacia quiénes o sobre qué recae el control social. ¿A la gestión misma? ¿A los funcionarios? ¿A los profesionales? ¿Al Estado? De acuerdo con los estudios realizados, dicho control se dio en los primeros años de funcionamiento de las CLAS. Probablemente se siga dando, pero este control solo recayó sobre los profesionales de la salud que fueron contratados bajo el régimen privado. Sus ingresos eran muy bajos y se les exigía más de ocho horas de trabajo. Este problema terminó cuando ellos se organizaron y lograron su nombramiento, retirándose muchos de las CLAS por considerar un abuso el trato que recibían y el régimen laboral al que estuvieron sometidos.

El control también se ha evidenciado hacia los funcionarios en algunos casos como, por ejemplo, en la CLAS Julio C. Tello. Aquí se dieron opiniones como la siguiente: Lo malo [de la CLAS] es que, si a la comunidad no le caes bien, te sacan. Si la asamblea considera que la doctora no es buena, tengan razón o no, la sacan (Portocarrero, 2006, p.198). La CLAS Laura Caller se inicia cuando una promotora de salud observó maltratos contra los usuarios. Ella se organizó con otras mujeres promotoras y consiguieron convertir en CLAS al centro de salud. Desde entonces, la promotora ha permanecido por ańos como presidenta de la CLAS. Damos por hecho que se ejercita el control hacia funcionarios; aun cuando sea parcial, cada asociación tiene sus particularidades. 
Hechos contrarios se han identificado donde son los gerentes los encargados de todo, o sea sin participación de la comunidad. Por ejemplo, en las CLAS La Querencia y Santa María de Huachipa, la comunidad ha delegado la autoridad a la Médicas Jefes porque confían en ellas y porque hay propietarios de tierras que aportan económicamente a las dos asociaciones (Portocarrero, 2006, p.198).

En todo caso, no se considera participación comunitaria a la participación de los propietarios que apoyan económicamente a las CLAS, porque no representan a la comunidad. Como se deduce, el control por parte de los miembros de la asamblea de las CLAS que representan a la comunidad se da, de alguna forma, específicamente en los factores desempeño y calidad del trato hacia los usuarios por parte del personal del MINSA. ¿Pero quiénes controlan la gestión de las CLAS? La comunidad no lo hace porque no participa. Como se ha visto en todas las investigaciones, esta no conoce qué es ni tampoco cómo funciona una CLAS.

Definitivamente no se ha dado el control social como estrategia de política diseñada en el gobierno de Alberto Fujimori. Menos aún corresponde a un Estado democrático de derecho de acuerdo con la Constitución Política del Perú. ¿Por qué consideramos que no se ha dado dicho control social? A nuestro criterio, porque desde la creación de las CLAS se proyectó una participación comunitaria ajena a esta función. Otro aspecto, todos los elementos de la propuesta fueron decretados anticipadamente por el Estado a través del MINSA, no por la comunidad que debía cogestionar los servicios de salud en el marco de la modernización del Estado y la nueva gestión pública. El modelo CLAS se concibe y desarrolla como una empresa donde los accionistas son los representantes de la comunidad, que, en realidad, por la forma de elección ya explicada y el papel del Estado, no lo son. Portocarrero (2006) es muy claro cuando dice:

$\mathrm{Al}$ convertirse en una asociación CLAS, el establecimiento de salud adquiere personalidad jurídica como entidad privada sin fines de lucro, lo que le otorga autonomía respecto al MINSA para establecer convenios de cooperación con instituciones, recibir donaciones y administrar sus propios recursos. Esta transferencia de poder, sin embargo, está sujeta a una serie de obligaciones y derechos especificados en el contrato de Administración Compartida. (p.173)

Esta es la forma como el Estado se sustrae de su responsabilidad con la salud de los más pobres, quienes tienen que resolver sus problemas de salud valiéndose de donaciones y recursos propios.

El éxito de estas asociaciones, en determinado momento, se dio porque la comunidad por lo menos recibía atención con un mejor trato y oportunamente. Esto gracias al control de los miembros de la CLAS sobre los funcionarios y profesionales involucrados, aun cuando la comunidad no los eligió democráticamente. Actualmente existen las CLAS en un menor número en relación a otros años. Con la Ley 29124, promulgada en el gobierno de Alan García, se ha tratado de organizar a las CLAS con las regiones y gobiernos locales; tarea aún inconclusa. Puede decirse que a la fecha no se ha logrado la organización. Son necesarios otros estudios para evaluar una experiencia que es muy importante con miras a su mejoramiento futuro.

\section{Conclusiones}

La participación de la comunidad sí es un elemento fundamental en la aplicación de las políticas de salud, toda vez que nadie mejor que la propia población para identificar sus problemas de salud y velar por su atención adecuada.

Los derechos a participar democráticamente en las políticas de salud están limitados por el propio Estado, tanto en el diseño como en su implementación, en la mayoría de los países de América Latina.

Pese a que la participación democrática está presente, no solo en el discurso de los gobernantes, sino también en sendos documentos oficiales y acuerdos internacionales, esta participación está limitada a los objetivos económicos y políticos del Estado.

La política focalizada de salud en el Perú expresada en el sistema CLAS, aún vigente, no cuenta con mecanismos de promoción para el ejercicio democrático de la población.

En todo el Perú, las CLAS tienen problemas comunes de representatividad, su carácter privado, y forma de elección controlada atenta contra los de- 
rechos ciudadanos de libertad y autonomía frente al Estado.

Se comprueba que en los países atrasados aún no se promueve la participación ciudadana, la mejora vista como mayor organización, desarrollo de capital social y control ciudadano en la implementación de las políticas de salud está ausente.

Esclarecer a la comunidad, sobre todo a los más necesitados, la importancia de su participación en pro de su bienestar es una obligación de la academia, como parte de la responsabilidad social que compete en este caso a la UNMSM.

El modelo CLAS no debe desaparecer, pero sí se tiene que fortalecer la participación ciudadana, los pobladores solicitan mayor atención del Estado.

\section{Referencias bibliográficas}

Arévalo, D. A. (2004). Participación Comunitaria y Control Social en el Sistema de Salud. Revista Salud pública, 6(2), 107-139.

Arroyo, J. (2001). Gobernabilidad en salud en el Perú en los 90. Lima: Ministerio de Salud del Perú.

AlbúJAR, P. J. (2016). El proceso de participación comunitaria de las CLAS como promotor de ciudadanía. Estudio de caso de la CLAS de Miraflores alto de Chimbote. Tesis para optar el grado de Magíster en Gerencia Social. Lima: Pontificia Universidad Católica del Perú.

Frisancho, A. D. (2005). Reforma del Estado y de la Administración Pública. Documento presentado en el $\mathrm{X}$ Congreso Internacional del CLAD sobre la Reforma del Estado y de la Administración Pública, Santiago, de 18 al 21 de octubre.

Bettina, A. (2005). El desarrollismo y la Reconceptualización. ¿Contraposición o avances para una nueva realidad? Revista Katálysis, 8(2), 193-198.

Bowyer, T. J. (2004). Participación Popular y el Estado. Lima: Instituto de estudios Latinoamericanos. Escuela de Estudios Avanzados. Universidad de Londres.

Cortez, R. y Phumpiu, P. (1999). La entrega de servicios de salud en los Centros de Administración Compartida (CLAS): El caso Perú. Lima: Centro de Investigaciones de la Universidad del Pacífico.

Salud Pública de México (1978). Informe de la Conferencia Internacional de Atención Primaria de
Salud (Época V, Volumen XX, Número 6). Recuperado de:http://saludpublica.mx/index.php/spm/article/view 1986/972

DíAz, R. (2001). CLAS: Una experiencia para descentralizar servicios de salud. Semanario Gestión Médica, 226(6), 18-19.

Gómez, E. (2008). Geopolítica del Desarrollo Comunitario: Reflexiones para Trabajo Social. Revista Ra Ximbai, 4(3), 519-542.

Huerta, M. G. (2005). El neoliberalismo y la conformación del Estado subsidiario. Politica y Cultura, 24, 121-150.

Laurell, A. C. (1994). La salud de derecho social a mercancía. En: Laurell, A. C. (coord.), Nuevas Tendencias y Alternativas en el Sector Salud (pp. 9-32). México D.-F., México, Fundación Friedrich Ebert \& UAM.

Ministerio de Salud (1996). Los Comités Locales de Administración en Salud (CLAS) - Organización y Modelo de Gestión "El Programa de Salud Local». Lima: Ministerio de Salud del Perú \& UNICEF.

Ocampo, J. (2008). Paulo Freire y la Pedagogía del Oprimido. Revista Rhela, 10, 57-72.

OAKLEY, P. (1990). Intervención de la comunidad en el desarrollo sanitario. Ginebra, Suiza: Organización Mundial de la Salud.

Portocarrero, F. et al. (2006). Capital social y democracia. Lima: Centro de investigación de la Universidad del Pacífico.

Prieto, R. y Wernerus, T. (2018). Plan nacional de formación. Control Social a la gestión pública. Bogotá, Colombia: Departamento Administrativo de la Función Pública.

Briceño-León, R. (1998). El contexto político de la participación comunitaria en América Latina. Revista Saúde Pública, 14(2), 141-147.

Salazar, A. (2015). Mecanismos de control social a la gestión pública nacional, regional y local. Análisis comparado con otros países de Sudamérica. Actualidad Gubernamental, 78(3), 4-6.

ZaKus, D. (1988). La participación comunitaria en los programas de atención primaria a la salud en el Tercer Mundo. Revista Salud Pública, 30(2), 151-174.

ZicCARDI, A. (1999). Los actores de la participación ciudadana. Instituto de Investigaciones Sociales, UNAM, México. Recuperado de: https://controlatugobierno. $\mathrm{com} /$ archivos/bibliografia/ziccardiactoresparticipacion.pdf 